\title{
Real-time Intelligent PID Controller for Ultrasonic Motor
}

\author{
Shenglin $\mathrm{Mu}^{\mathrm{a},{ }^{*},}$ Kanya Tanaka ${ }^{\mathrm{b}}$, Shota Nakashima ${ }^{\mathrm{b}}$, Djoewahir Alrijadjis ${ }^{\mathrm{b}}$ \\ ${ }^{\text {a }}$ Department of Electronic Control Engineering, Hiroshima National College of Maritime Technology, 4272-1, \\ Higashino, Osakikamijima-cho, Toyota-gun, Hiroshima 725-0231, Japan \\ ${ }^{\mathrm{b}}$ Graduate School of Science and Engineering, Yamaguchi University, Ube, Yamaguchi, 755-8611, Japan \\ *Corresponding Author: mshenglin@ @iroshima-cmt.ac.jp
}

\begin{abstract}
In order to get good performance of ultrasonic motors (USMs) in real applications, a real-time intelligent PID controller is proposed in this paper. To overcome the problems of characteristic variation and nonlinearity, an intelligent PID controller combined with particle swarm optimization (PSO) type neural network (NN) is studied in real-time environment for USM control. In the proposed method, an NN controller is designed for adjusting PID gains. The learning of NN is implemented by PSO updating the weights of NN on-line. By employing the proposed method, the characteristic changes and nonlinearity of USM can be compensated effectively in real-time environment. The effectiveness of the method is confirmed by experiments.
\end{abstract}

Keywords: ultrasonic motor, PID control, real-time, neural network, particle swarm optimization.

\section{Introduction}

As industrial technology develops in recent years, for actuators in industrial applications, there are more and more high requirements. In order to get more and more excellent features, many kinds of novel actuators have been developing in recent decades. Among the actuators, ultrasonic motors (USMs), as a novel kind of actuators with perspective, are quickly developed. They are designed, improved and applied for meeting new and special requirements in many specific applications ${ }^{(1-3)}$. As we see in Fig. 1, it is the cutaway view of traveling wave ultrasonic motor (TWUSM). USMs are friction driven electronic motors. The reason why this kind of actuators is so widely used is that there are many excellent characteristics of USMs comparing with conventional electromagnetic motors, such as, quick response, noiseless, compactness, low speed, high torque, , no magnetism and so on. In order to make use of the excellent features, many researches have been implemented.

Especially, in the field of USM control, there are many methods developed in recent years for compensating characteristic changes and nonlinearity ${ }^{(4)}{ }^{(4)}$. T. Senjyu et al. proposed a direct neural network (NN) controller for the position control of USM. In the method, a three-layer NN controller using back-propagation (BP) method has been reported ${ }^{(5)}$. A control scheme using NN for USM has been proposed by F. Lin et al. in Ref. (6). In the control scheme, the robust control system based on Lyapunov function using two NNs is designed to control the rotor position of USM. Different from previous research employing BP methods, the intelligent internal model control (IMC) type PID employing particle swarm optimization (PSO) has been studied in our previous research ${ }^{(7)}$. It is certified that intelligent control based on PID theory using NN is able to compensate characteristic changes and nonlinearity of USM in real applications.

In this paper, a real-time intelligent PID controller is reported as an extension of previous research. The intelligent method of PSO type NN is designed for tuning gains in PID

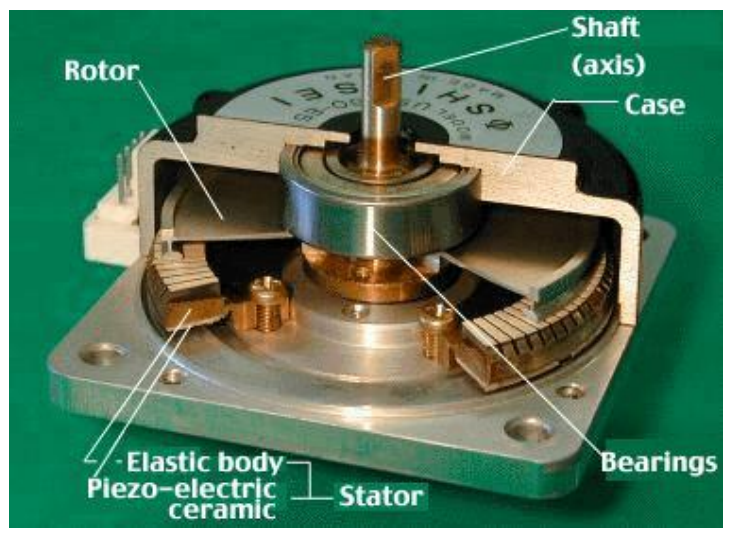

Fig. 1. Cutaway view of TWUSM. 
controller in USM servo system. The learning of NN is carried on by PSO updating weights on-line. The paper is organized as follows. After this introduce section, the proposed intelligent PID controller is introduced in Sect. 2. USM servo system, the real-time experimental environment is introduced in Sect. 3. In Sect. 4, the experimental results are studied. Section 5 gives conclusions.

\section{Intelligent PID Controller}

\subsection{PID Control for USM}

Since PID control is quite simple and effective, it is considered as the most widely used control scheme in industrial fields ${ }^{(8)}$. In our proposed method, PID control is applied as the direct controller. Figure 2 shows the basic scheme of PID control for USM. In the scheme, $r(k), u(k)$, and $y(k)$ are the object input, the input and the output, respectively. $G_{P I D}\left(z^{-1}\right)$ is the PID controller. The input $u(k)$ is synthesized as

$$
\begin{aligned}
& u(k)=u(k-1)+\left(K_{P}+K_{I}+K_{D}\right) e(k) \\
& -\left(K_{P}+2 K_{D}\right) e(k-1)+K_{D} e(k-2)
\end{aligned}
$$

where $K_{P}, K_{I}$ and $K_{D}$ are the gains of the PID controller. By tuning the PID gains, the control performance can be adjusted. In the scheme, the error denoted by $e(k)$ expressed as

$$
e(k)=r(k)-y(k)
$$

The PID controller $G_{P I D}\left(z^{-1}\right)$ can be denoted as Eq. (3) shows.

$$
G_{P I D}\left(z^{-1}\right)=\frac{K_{P}\left(1-z^{-1}\right)+K_{I}+K_{D}\left(1-z^{-1}\right)^{2}}{1-z^{-1}}
$$

Theoretically, we can get good control performance by making fine tuning of the PID gains in USM control. However, since the USM is a non-linear plant without mathematical model, the fine tuning is difficult. Meanwhile, characteristics of USM varies according to running conditions. Conventional fixed gain type PID controller cannot deal with the characteristics of USM ${ }^{(9,10)}$. For

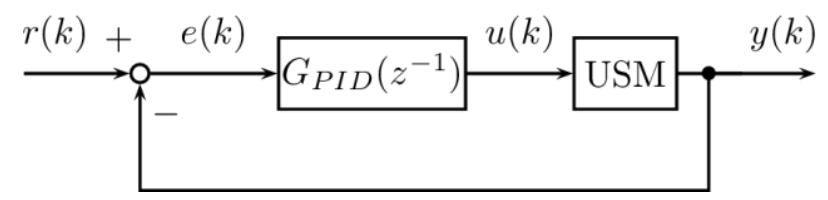

Fig. 2. Block diagram of PID control for USM.

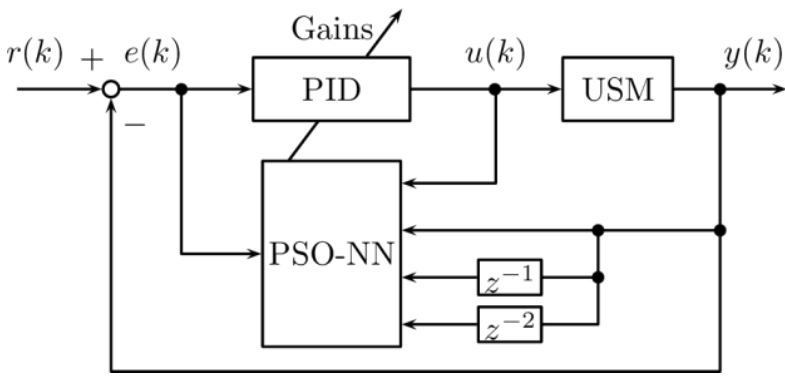

Fig. 3. Control scheme of proposed method.

obtaining good performance in USM control, gains of PID controller requires continuous adjustment according to characteristic changes. In this research, the prosed intelligent method is designed as Fig. 3 shows. Based on conventional PID controller for USM, the intelligent model - PSO type NN is employed for updating the PID gains on-line. As we see in the scheme, the input of the intelligent controller is the discrete time signal of $[u(k-1), y(k), y(k-1), y(k-2)]$. The output is the PID gains. The scheme is designed for tuning PID gains automatically to achieve good performance in USM control.

\subsection{PSO Type Neural Network}

In this section, the intelligent unit combined with PSO and $\mathrm{NN}$ is illustrated. As we know that $\mathrm{NN}$ is one of important algorithms in artificial intelligence, which is developed so fast in recent decades that some NN models have been applied in industrial products. Usually, NN model is constructed by connections of neurons, which are designed according to the biological neural system. Because of the features of $\mathrm{NN}$, as an important optimization tool $\mathrm{NN}$ is widely applied in many aspects, such as the optimal or adaptive control engineering, pattern matching technique in financial field and traffic and communication fields. Especially, NN is considered as an important tool to contribute a lot in the control of non-linear plants. In our research, NN is applied for updating the PID gains in proposed scheme for compensating nonlinearity in USM control. The NN scheme we propose is a fixed structure. The learning is implemented by PSO algorithm.

On the other hand, PSO is a global optimization algorithm discovered through the simulation of a simplified social model. Since it is simple in concept and easy to implement, it is quite attractive in the field of probability optimization in recent years ${ }^{(11-14)}$. Figure 4 shows an example of particle movement of PSO. $x_{i}(k)$ means the position of the $i_{\text {th }}$ particle in the $k$ step, $x_{i}(k-1)$ and $x_{i}(k+1)$ mean the position of the $(k-1)$ step and the $(k+1)$ step, 


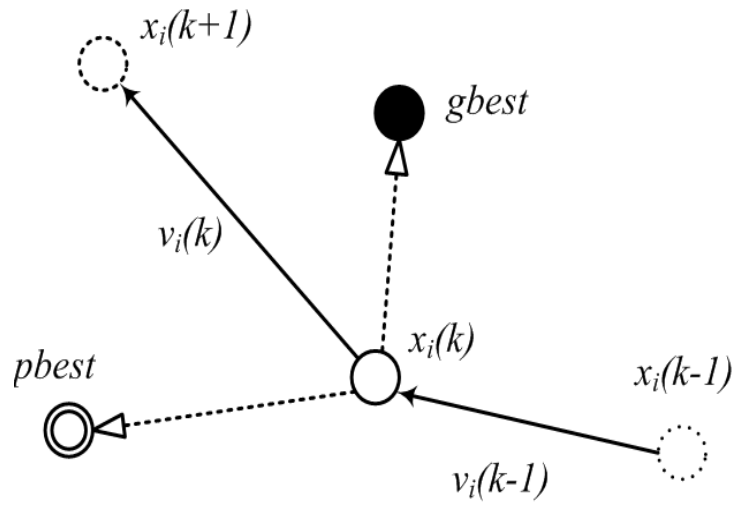

Fig. 4. Illustration of particle movement in PSO.

respectively. gbest is the best position of the whole swarm, and pbest is the best position of the particle. $v_{i}(k)$, which expresses the position variance, is the velocity of the particle at the $k$ step. It is related to the best positions as the dash lines show in the figure. In NN learning, we define the value of weights as the position information contained by $N$ particles. The weights of NN are designed to be updated by an inertia weight approach (IWA) type PSO algorithm expressed in Eq.(4) and Eq.(5).

$$
\begin{aligned}
& V_{n+1}=\tau \cdot V_{n}+c_{1} \cdot r_{1} \cdot\left(\text { pbest }_{n}-X_{n}\right) \\
& +c_{2} \cdot r_{2} \cdot\left(\text { gbest }_{n}-X_{n}\right) \\
& X_{n+1}=X_{n}+V_{n}
\end{aligned}
$$

In Eq.(4), $c_{1}$ and $c_{2}$ are two positive constants, $r_{1}$ and $r_{2}$ are two random numbers within [0,1]. In Eq.(5), the weight factor which balances the global and local search, is calculated as follows.

$$
\tau=\tau_{\max }-\frac{\tau_{\max }-\tau_{\min }}{\text { episode } d_{\text {max }}} \cdot \text { episoc }
$$

where episode means the number of current iteration, episode $_{\text {max }}$ presents the max iteration number in the searching. $\tau_{\max }$ and $\tau_{\min }$ are the weight factor's maximum and minimum value, respectively. As we see in Fig. 5, it is the flowchart of the proposed method. The learning of PSO-NN and updating of PID controller is designed in the simple loop as the figure shows. More details about the specifications of PSO type NN algorithm for experiment is to be introduced in Sect.4.

\section{USM Servo System and Experiments}

In this section, the USM servo system is presented. In the USM servo system, the motor, encoder, and the magnetic brake are connected on a common axis as shown in Fig. 6. The position signal fetched by the encoder is input to the counter board connected to the PC. Information of the control input calculated from the output and the object input in the PC is transferred to the driving circuit via the $\mathrm{I} / \mathrm{O}$ board. The frequency control is applied in this servo system. High frequency makes the motor controlled with high accuracy. The driving frequency will vary from $42.5 \mathrm{kHz}$ to $46.0 \mathrm{kHz}$ higher than resonance frequency of the USM.

In order to confirm the effectiveness of the proposed method, a group of experiments are implemented in the USM

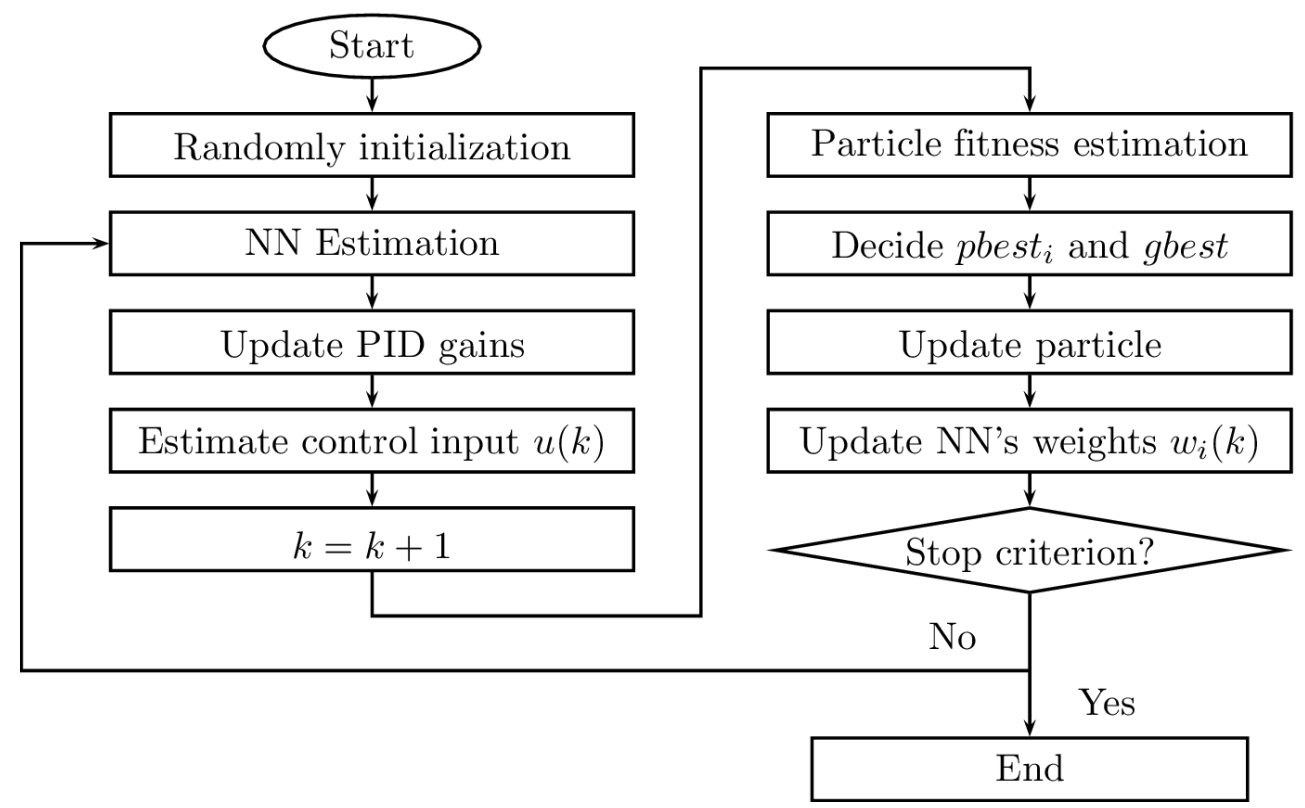

Fig. 5. Flowchart of proposed method. 


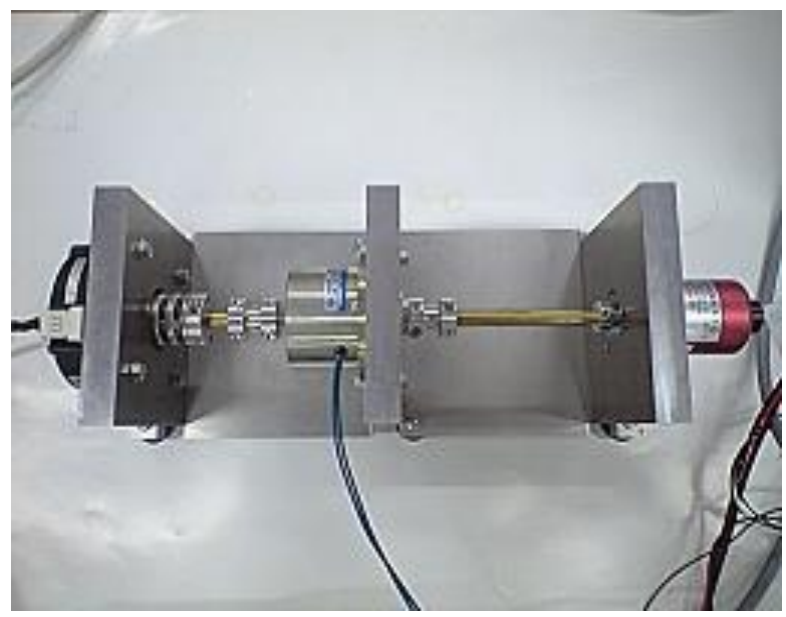

Fig. 6. Photograph of USM servo system.

servo system. The purpose of the experiments is to validate the performance of this proposed method in real-time control. The object input $r(k)$ is a sine signal. The amplitude is set from +45 deg. to -45 deg. The period of the sine wave is 2 second. Swarm of PSO with 10 particles was designed for updating $\mathrm{NN}$ weights in this intelligent method. All the particles are initialized by random numbers from -1 to 1 . Learning of NN, that is calculation of PSO, the inertia weight factors $\tau_{\max }$ and $\tau_{\min }$ are set as $\tau_{\max }=0.9$ and $\tau_{\min }=0.4$, respectively. The positive constants $c_{1}$ and $c_{2}$ are set as $c_{1}=c_{2}=2.0$. The particles are designed to be evaluated by the following function with the real-time error signal $e(k)$.

$$
\text { fitness }=\frac{1}{1+e(k)^{2}}
$$

In the experiments, the sine wave signal is set to run 20 cycles. Figure 7 shows the response of the first 3 cycle of the proposed method in real-time control. We can see that the output follows the object input in the run. Figure 8 shows the variation of PID gains in the real-time control, it is clear that the proposed method is able to get proper gains in real-time compensating. It can be stated that the USM has the different characteristic according to the rotatory direction, and that the proposed method is capable to search optimal value in realtime control. Meanwhile, it can be concluded that PSO is an effective tool to be combined with $\mathrm{NN}$ in real-time applications.

\section{Conclusions}

In this paper, a real-time intelligent PID control scheme combined with PSO type NN has been proposed for USM.

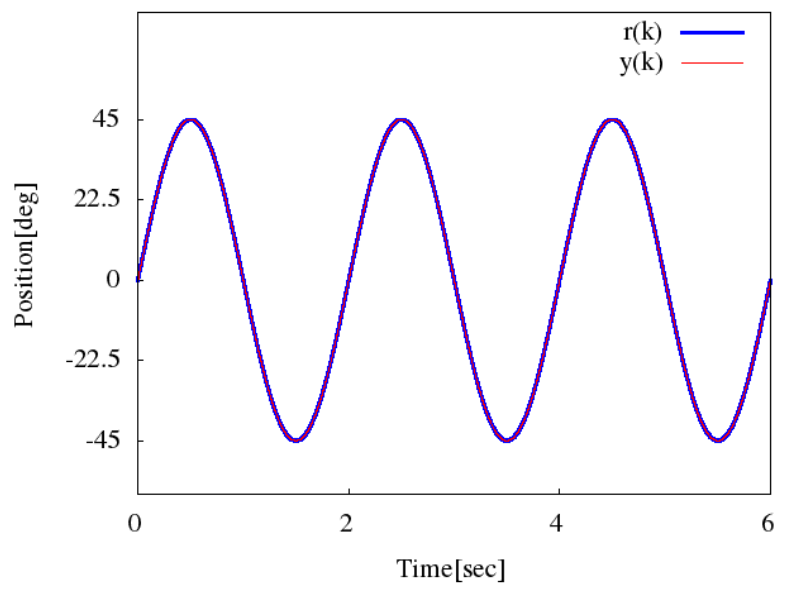

Fig. 7. Response in real-time control.

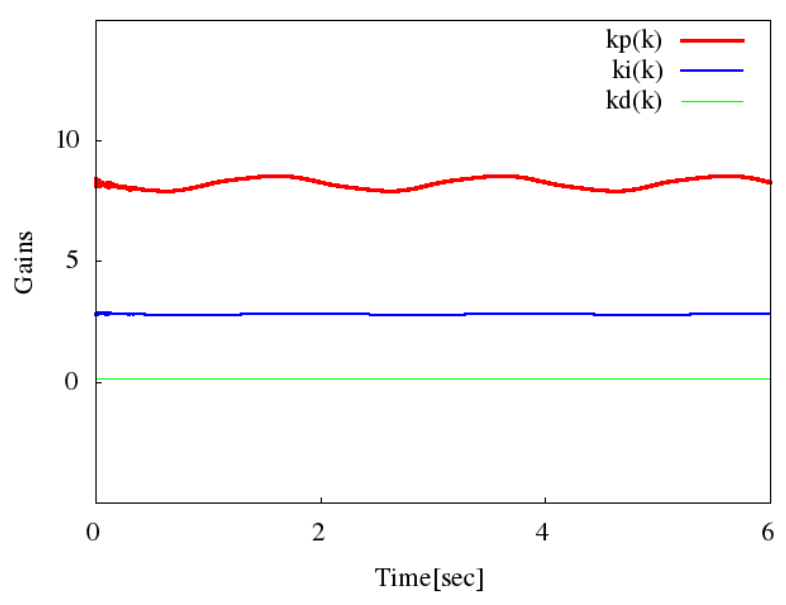

(b) Proposed method.

Fig. 8. Variation of gains in real-time control.

$\mathrm{NN}$ is employed for optimizing the gains in PID controller. PSO algorithm is applied in NN's learning. The weights of NN is updated by PSO on-line. The proposed method is able to be applied in compensating characteristic changes USM in real-time control. The effectiveness of the proposed method is confirmed by experiments using USM servo system.

\section{References}

(1) T. Kenjo and T. Sashida: "An Introduction of Ultrasonic Motor" Oxford Science Publications, 1993.

(2) K. Uchino: "Piezoelectric ultrasonic motors: Overview", Smart Material Structure, Vol. 7: pp. 273-285, 1998

(3) K. Adachi: "Actuator with friction drive: Ultrasonic motor", The Japan Society of Mechanical Engineers, Vol. 108: pp. 48-51, 2005 
(4) C. Zhao: "Ultrasonic Motor - Technologies and Applications", Science Press Beijing and SpringerVerlag Berlin Heidelberg, Beijing, 2011

(5) T. Senjyu, H. Miyazato, and K. Uezato: "Position control of ultrasonic motor using neural network" (in Japanese), Transaction of the Institute of Electrical Engineers of Japan D, Vol.116: pp. 1059-1066, 1996

(6) F. Lin, R. Wai, and C. Hong: "Identification and control of rotary traveling-wave type ultrasonic motor using neural networks", IEEE Transactions on Control Systems Technology, Vol. 9: pp. 672-680, 2001

(7) S. Mu and K. Tanaka: "Intelligent IMC-PID control using PSO for ultrasonic motor", International Journal of Engineering Innovation and Management, Vol. 1: pp. 69-76, 2011

(8) K. Astrom, T. Hagglund, C. Hang, and W. Ho: "Automatic tuning and adaptation for PID controllers a survey”, Control Engineering Practice, Vol. 1: pp. 699-714, 1993

(9) S. Mu, K. Tanaka, Y. Wakasa, T. Akashi, N. Kobayashi, S. Uchikado, and Y. Osa: "Intelligent IMC-PID control for ultrasonic motor", Proceedings of International Conference of Networking, Sensing, and Control, 2009

(10)K. Tanaka, Y. Yoshimura, Y. Wakasa, T. Akashi, M. Oka, and S. Mu: "Variable gain type intelligent PID control for ultrasonic motor" (in Japanese), The Japan Society Applied Electromagnetics and Mechanics, Vol. 17: pp. 107-113, 2009

(11)J. Kennedy and R. Eberhart: "Particle Swarm Optimization", Proc. IEEE Int. Conf. Neural Networks, Perth, Australia, pp. 1942-1948, 1995

(12)M. Clerc and J. Kennedy: "The Particle Swarm: Explosion, Stability, and Convergence in a MultiDimensional Complex Space", IEEE Trans. Evolutionary Computation, Vol. 6, No. 1, pp.58-73, 2002

(13)J. Kennedy, R. Eberhart: “Swarm Intelligence”, Morgan Kaufmann Publishers, 2001

(14) M. Ito and M. Tanaka: “A Study of Particle Swarm Optimization for Neural Network Training", A publication of Electronics, Information and System Society, pp. 1087-1089, 2005 